\title{
Neural Network Estimation of Generator Slot Fields for the Calculation of Radial Field Losses and Circulating Currents
}

\author{
E. Schlemmer, B. Streibl, and F. Müller \\ VA TECH HYDRO GmbH \& Co \\ Elingasse 3, A-8160 Weiz (Austria) \\ Phone +43 31726062812 , Fax +43 31726062098 \\ e-mail: Erwin.Schlemmer@vatech-hydro.at
}

\begin{abstract}
In this paper, an approximate method for the fast calculation of magnetic fields inside the stator slots of large hydro generators is presented. Due to its relatively low computational cost, the approach is suitable for every-day-use calculations in the process of tendering and design of hydro generators. The scheme comprises two parts. The first one is a Boundary Element Method (BEM) solution of a slot with current-carrying strands and ideally permeable walls. The second part consists in an Artificial Neural Network (ANN) for the incorporation of secondary effects, such as tooth saturation. The effects of induced eddy currents on the flux density at the strands' centres are taken into account by an iterative BEM solution with successive corrections of the resulting fields. Since eddy current effects are comparably small for the configurations usually applied, convergence is readily achieved. The training data for the ANN were obtained from a comparison between the linear BEM results and Finite Element (FE) computations which have been carried out on a set of 65 hydro generators at different slot temperatures. By employing the BEM approach, which captures most of the underlying physics, and a corrective neural network, a grey box model is obtained. This has the advantage that the output of the net is "safeguarded" by the BEM solution. In order to get a robust representation of the data, the training of the ANN was carried out applying a Genetic Algorithm (GA).
\end{abstract}

\section{Key words}

Finite element methods, boundary element methods, synchronous generators, radial field losses, genetic algorithms, and artificial neural networks.

\section{Introduction}

The calculation of circulating currents and radial field losses in the stator bars of large generators has been dealt with by several authors during the last decades, e.g. [1][10]. Circulating current losses have their origin in the uneven distribution of the strand currents in a stator bar. This is due to induced voltages between the individual strands of a bar. One part of the voltages originates from the stray field in the end winding region, the other part from the slot field in the stator section. The calculation methods for circulating currents are based on the approach of [5], [6]. It consists of an axial subdivision of the strands in order to model the transposition (Fig. 1) by suitable displacements of the parts along the bar.

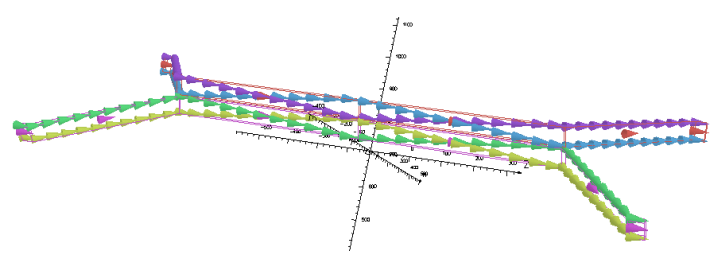

Fig. 1. Discrete model of a Roebel bar.

Within the stator slots, the mutual interactions of the strands are captured by an impedance model. In the end region, however, the whole end winding contributes to the stray fields (with distance as an ameliorating effect). Nevertheless, in most approaches, Biot-Savart integration in the end region is employed where the boundary conditions are enforced by suitably chosen mirror conductors (Fig. 2).

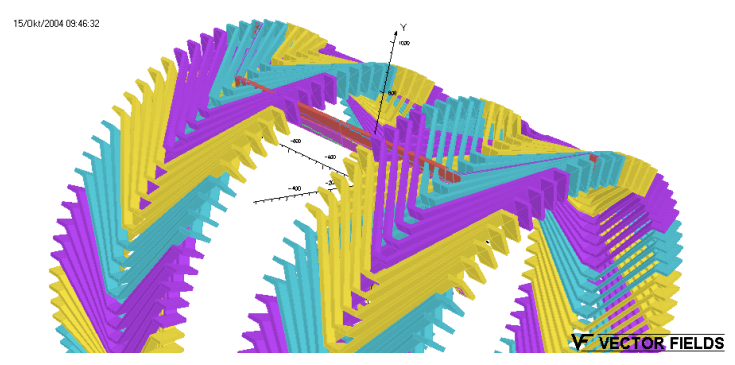

Fig. 2. Conductor model of both end winding regions for BiotSavart integration.

Transpositions for keeping circulating current losses low are well known and need not be dwelt on here. 
Nevertheless, it is of utmost importance to pre-calculate the effect of a given transposition on the overall losses of a generator. However, some of the most decisive influence factors, i.e. the impedances of the strands within the slot region, are often based on a linear and unsaturated slot model.

During the last years, significant improvements have been achieved on this field. In [3] and [4], conformal mapping and FE-based slot fields have been employed. The availability of radial slot fields is a considerable advantage, especially in the case of large turbine generators with double Roebel bars or directly cooled stator windings.

Good estimations of the radial slot fields give the possibility to calculate the radial field losses [7]. Although the use of 2d-FE would give relatively good answers for the fields, it is hardly ever employed in every-day-use. A full 3d-FE model with bar transposition is known from the literature, [8], but it seems to be of limited use in practice for reasons of high complexity.

On the other hand, the technique of conformal mapping has been employed for the solution of the slot opening problem for many years (e.g. [13] and [16]). Though this method gives good estimations for the radial fields near the slot opening, it is basically restricted to potential problems. That is, the slot walls are modelled as Dirichlet boundary conditions. The inclusion of sources (i.e. strand currents) has rarely been attempted (e.g. [17]) because the sources have also to be transformed into the $w$-plane which is a quite formidable task.

Therefore, the findings of [1] - [4], [7] have been used as the basis for an approximation of the radial slot fields including tooth saturation and load. The basic physics of the problem is described using a BEM solution; corrections for non-linear effects are made by an ANN. The training data for the ANN have been found using FE results. However, due to the complexity of a $2 \mathrm{~d}$ rotational FE solution (Fig. 3), certain simplifications had to be made.

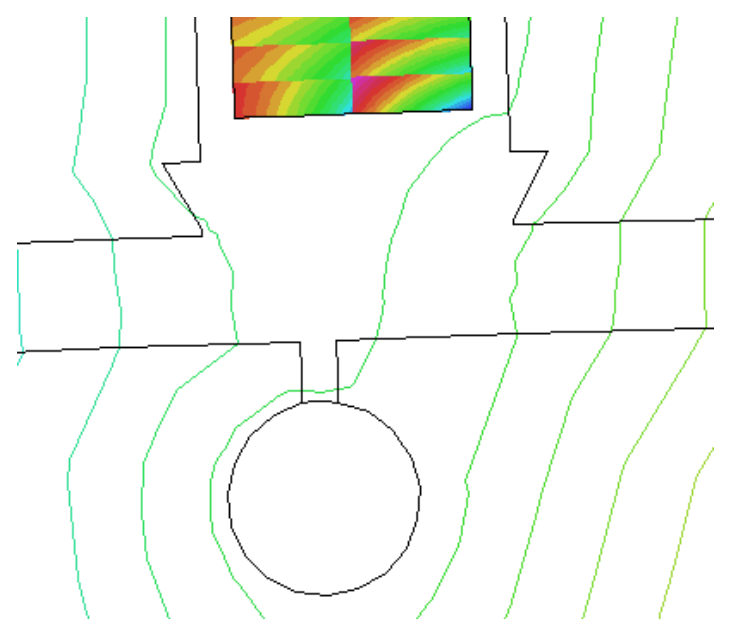

Fig. 3. Calculated stator strand currents due to radial fields at rated load.

First, the effects of rotor motion are neglected. They are replaced by an investigation of a single slot under harmonic excitation. This does also imply that losses of current harmonics are not taken into account. From the findings in [7], this seems to be justified. Second, the potential distribution along the boundaries of the single slot model had to be approximated by functional boundary conditions. These were derived from flux patterns that had been obtained from $2 \mathrm{~d}$ rotational FE. Although these assumptions appear to be rather far reaching at the first glance, they nevertheless give good answers when compared with rotational FE. Moreover, with somewhat higher effort, one could also derive training data from $2 \mathrm{~d}$ rotational FE models. This would enhance the accuracy further, albeit at rather high computational cost.

\section{Approximation of the Slot Field}

\section{A. Boundary Element Approach}

For a two dimensional (2d) approximation of the magnetic field inside a slot with ideally permeable walls, we start with the quasi-static $\operatorname{curl}(\mathbf{H})$ equation.

$\nabla \times \mathrm{H}=\mathrm{J}$

$\mathbf{J}$ comprises the net current of the strand as well as the eddy currents. Since the magnetic flux density $\mathbf{B}$ is solenoidal,

$\nabla \cdot \mathrm{B}=0$

it can be expressed by the curl of the axial $(z-)$ component of a magnetic vector potential $\mathbf{A}$.

$\mathrm{B}=\nabla \times \mathrm{A}=\nabla \times A_{z} 1_{z}$

Together with the material law for free space

$\mathrm{B}=\mu_{0} \mathrm{H}$

Poisson's differential equation can be derived for the $z$ component of the magnetic vector potential in a $2 \mathrm{~d}$ problem.

$\nabla^{2} A_{z}=\Delta A_{z}=-\mu_{0} J_{z}$

Contrary to [10], fast transients are not of interest here. Therefore, a static approach with successive iterations for the allowance of induced eddy currents was chosen.

Following [11], the magnetic flux density is split into two parts, $\mathbf{B}^{\propto}$ and $\mathbf{B}^{D}$, where $\mathbf{B}^{\propto}$ is the homogeneous flux across the air gap and $\mathbf{B}^{D}$ the disturbance flux caused by the presence of slot walls and current carrying conductors inside the slots.

$\mathrm{B}=\mathrm{B}^{\infty}+\mathrm{B}^{D}$

Whereas $\mathbf{B}^{\propto}$ is given in closed form, $\mathbf{B}^{D}$ is expressed as the curl of two vector potentials $A_{z}^{W}+A_{z}^{C}$, where the first one takes the influence of the walls into account and the second one the currents inside the slot.

$\mathrm{B}=\mathrm{B}^{\infty}+\nabla \times\left(A_{z}^{W}+A_{z}^{C}\right) 1_{z}$ 
Since the slot walls are thought as infinitely permeable, the (pseudo-) tangential component of $\mathbf{B}$ is set to zero along those slot walls.

$\mathrm{n} \times \mathrm{B}=\mathrm{n} \times\left(\mathrm{B}^{\infty}+\nabla \times\left(A_{z}^{W}+A_{z}^{C}\right) 1_{z}\right)=0$

Together with the normal derivative of the z-component of a vector potential in $2 \mathrm{~d}$,

$\mathrm{n} \times\left(\nabla \times A_{z} 1_{z}\right)=-\frac{\partial A_{z}}{\partial n}$,

the following expression is obtained for the normal derivate of the wall disturbance potential $A_{z}^{W}$.

$$
\begin{aligned}
\frac{\partial A_{z}^{W}}{\partial n}=\mathrm{n} \times B^{\infty}-\frac{\partial A_{z}^{C}}{\partial n}=\mathrm{n} \times B^{\infty}-\frac{\partial}{\partial n}\left(\mu_{0} \int_{\Omega} J_{z} G d \Omega\right)= \\
=\mathrm{n} \times B^{\infty}-\left(\mu_{0} \int_{\Omega} J_{z} \frac{\partial G}{\partial n} d \Omega\right)
\end{aligned}
$$

In (10), the Newtonian potential (11) of the currents for free space has been used. $\Omega$ is the domain of interest, i.e. slot and air gap. Since $J_{z}=0$ outside the strands, the integration is carried out over the conductors only. The normal derivative in (10) is applied with respect to observation point co-ordinates. The domain integral runs over source co-ordinates, only Green's function depends on both co-ordinate systems. Therefore, the derivation could be applied directly to Green's function.

$$
A_{z}^{C}=\mu_{0} \int_{\Omega} J_{z} G d \Omega
$$

The derivation of the boundary integral formulation (12) for the problem at hand can be found in textbooks, e.g. [11] - [14], and shall not be repeated here. Moreover, the spatial dependencies of Green's functions and the potentials have been tacitly omitted for the sake of brevity. In (12), $\Gamma$ is the boundary of $\Omega$. The disturbance potential $A_{z}^{W}$ has no sources within $\Omega$. In the second domain integral, the definition of Green's function has been introduced, where $\delta$ is Dirac's delta function.

$$
\begin{gathered}
\int_{\Omega}\left(G \Delta A_{z}^{W}-A_{z}^{W} \Delta G\right) d \Omega=\int_{\Omega}\left(G \cdot 0-A_{z}^{W}(-\delta)\right) d \Omega= \\
=A_{z}^{W}=\oint_{\Gamma}\left(A_{z}^{W} \frac{\partial G}{\partial n}-G \frac{\partial A_{z}^{W}}{\partial n}\right) d \Gamma
\end{gathered}
$$

When the point of observation approaches the boundary, the integral kernels become singular. The principal value evaluation of the boundary integrals leads to the integral equation (13) for the disturbance potential on the region boundaries. The normal derivative of the disturbance potential on the slot walls follows from (10).

$c A_{z}^{W}=\oint_{\Gamma}\left(A_{z}^{W} \frac{\partial G}{\partial n}-G \frac{\partial A_{z}^{W}}{\partial n}\right) d \Gamma$
In (13), $c$ is the coefficient of singularity which results from replacing the original boundary by a part of a circle around the coalescing observation and source points.

$c=\frac{\alpha}{2 \pi}$

On a smooth boundary, the value of $c$ is $1 / 2$. If the singularity is on a wedge with internal angle $\alpha$, the value of $c$ is determined by this angle divided by $2 \pi$.

The integral equation is solved by the Boundary Element Method (BEM). Details are not repeated here, because there is an ample choice of textbooks available on the topic, e.g. [11] or [12]. Even though it is not economical to derive equi-potentials or flux lines from a BEM solution, this has been done for the sake of comparison between BEM and FE results (Fig. 4). The flux lines in (a) have only been drawn in the air gap as it was not considered important to refine the underlying algorithms beyond measure.

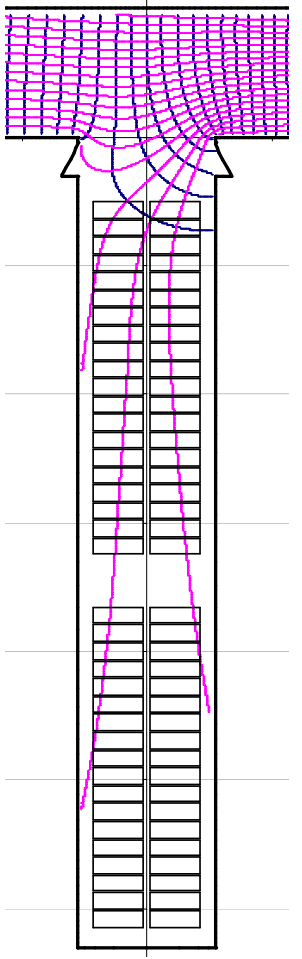

(a)

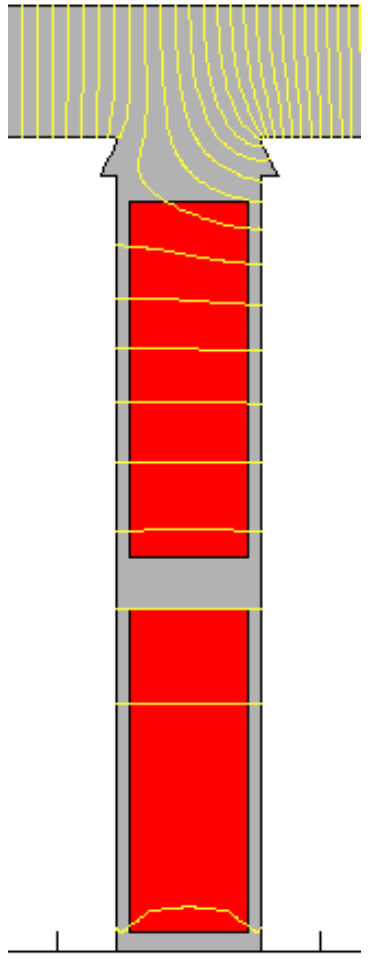

(b)
Fig. 4. Equi-potential and flux lines computed via the boundary representation (a) and from a FE solution (b).

The phenomena of fringing main flux and tooth tip singularity can be observed in Fig. 5. In the (thought) uppermost strands of the upper bar, the singularity at the tooth tip has a remarkable influence on the distribution of the magnetic flux density. The effect of the fringing main flux can also be seen clearly by the substantial $y$-directed field near the upper rim of the bar in (b). 


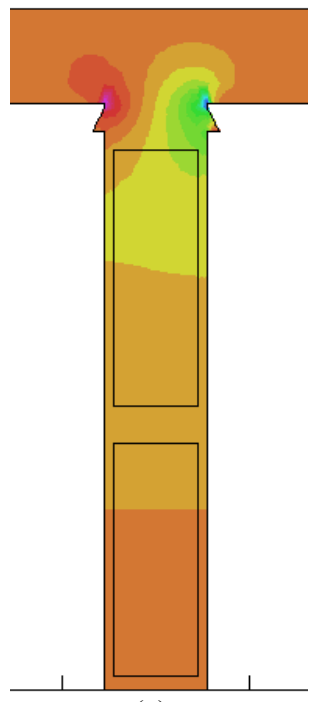

(a)

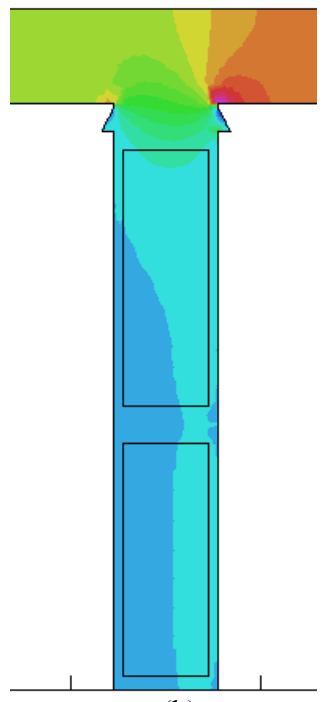

(b)
Fig. 5. Example of the distribution of the $x$-component (a) and of the $y$-component (b) of the flux density $\mathbf{B}$.

\section{B. Eddy Current Fields}

All quantities inside the slot are described in two parallel co-ordinate systems. First, the global $(x, y)$-system having its origin at the centre line at the slot opening. Second, there is a local $(\xi, \eta)$-system originating at every strand's centre (Fig. 6).

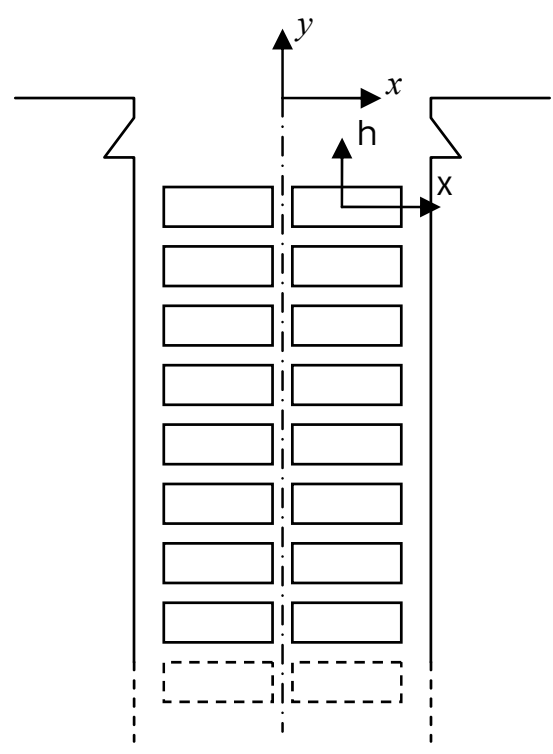

Fig. 6. Global (slot) and local (strand) co-ordinate systems.

For the representation of the magnetic flux density inside the conductors, the approach of [1], [2] is adopted to the situation given here. The flux density (15) is subdivided into two parts, i.e. a component $\mathbf{B}_{0}$ in the centre $\left(\xi_{m, i}, \eta_{m, i}\right)$ of strand $i$ and another component $\mathbf{B}_{C}$ which is due to the currents within this strand. The component $\mathbf{B}_{0}$ is determined mainly by the currents below the considered strands, the fringing main flux and by the singularities at the tooth tips. The contribution of the main flux due to saturation of the stator teeth is usually moderate (e.g. [7]) and can be captured by the neural network.

$$
\mathrm{B}_{i}(\xi, \eta)=\mathrm{B}_{0}\left(\xi_{m, i}, \eta_{m, i}\right)+\mathrm{B}_{C}(\xi, \eta)
$$

Since the dimensions of the conductors are roughly in the order of the skin depth, the spatial variation of the currents is assumed to be linear. Both the $x$ - and $y$ components of the magnetic flux set up eddy currents, which can be expressed as in (16). The strand width is denoted as $w, h$ stands for its height. $I$ symbolises the net current flowing in the strand.

$$
J_{z}(\xi, \eta)=\frac{I}{w h}+J_{z}\left(B_{y}, \xi\right)+J_{z}\left(B_{x}, \eta\right)
$$

In Fig. 7, these assumptions are made clearer. Since the loop for currents induced by $B_{y}$ is much wider than that for $B_{x}$, the respective $B_{y}$-currents will dominate. Currents set up by $B_{x}$ have a relatively modest influence on the overall field situation albeit they can create quite substantial losses. This is because $B_{x}$ increases steadily from the slot ground towards the opening. $B_{x}$ has typical values of about $0.3 \mathrm{~T}$ at the uppermost strands (see also Fig. 8). Consequently, many strands are exposed to relatively high $B_{x}$-values. The situation is contrary for $B_{y}$, since high values of $B_{y}$ are a local phenomenon near the slot opening.

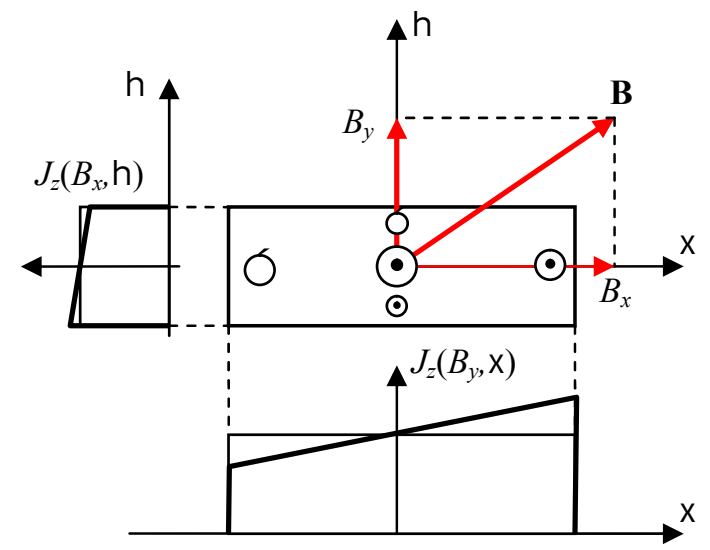

Fig. 7. Current density along $\eta=0$ and $\xi=0$.

Referring to Fig. 8, it can be observed that under idealised slot conditions (i.e. infinitely permeable walls, no fringing field, no influence of the tooth tips), the flux density resulting from a positive $z$-directed bar current is solely directed along the negative $x$-axis. Accordingly, the $y$-directed flux $B_{c, y}$ is zero, because it would have to pass a very long distance in air in front of an ideally permeable wall. In (17), this fact has been taken into account where $\omega$ is the angular frequency and $\sigma$ the conductivity of the strands.

$$
\begin{aligned}
J_{z}\left(B_{y}, \xi\right) & =-\frac{j \omega \sigma w}{12} B_{C, y}+j \omega \sigma\left(B_{0, y} \xi+B_{C, y} \frac{\xi^{2}}{w}\right) \approx \\
& \approx j \omega \sigma B_{0, y} \xi
\end{aligned}
$$


Also with reference to Fig. 8 , it can be stated that $B_{C, x}$ should not be neglected thus leading to (18).

$$
J_{z}\left(B_{x}, \eta\right)=\frac{j \omega \sigma h}{12} B_{C, x}-j \omega \sigma\left(B_{0, x} \eta+B_{C, x} \frac{\eta^{2}}{h}\right)
$$

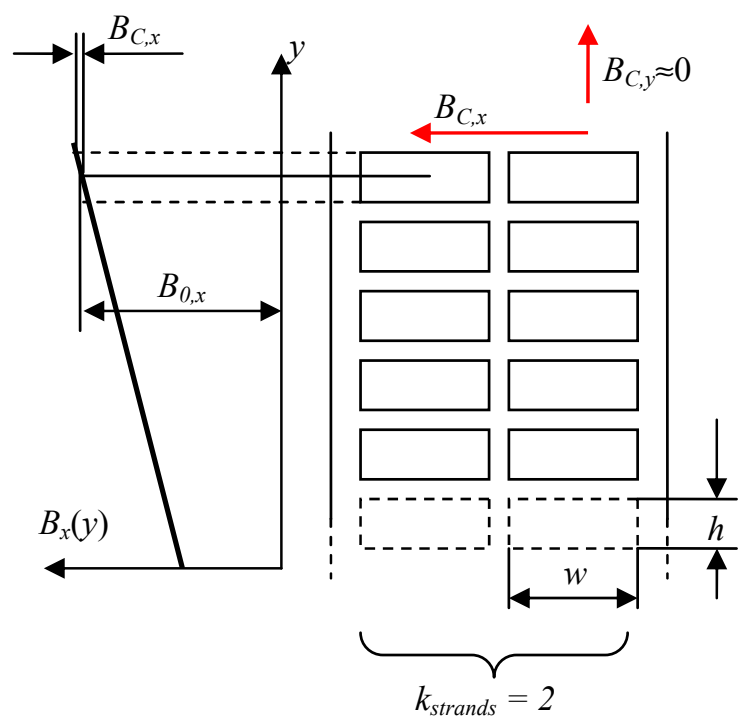

Fig. 8. Stray flux density inside an idealised slot. Only the upper part of the slot has been drawn.

From the conditions in the ideal slot and following the approach of [4], the magnitude of $B_{C, x}$ is calculated approximately via (19)

$$
B_{C, x} \approx-\mu_{0} \frac{I}{2 \beta}=-\mu_{0} \frac{I}{2 \frac{w_{\text {slot }}}{k_{\text {strands }}}},
$$

where $\beta$ is the part of the slot width which one of $k_{\text {strands }}$ parallel strands has to excite with its current $I$. Inserting (19) into (18), $B_{C, x}$ can be eliminated thus giving (20) for the expression of the current density due to the $x$-directed flux density.

$$
J_{z}\left(B_{x}, \eta\right) \approx I \cdot\left[\frac{j \omega \sigma \mu_{0}}{2 \beta}\left(\frac{h}{12}-\frac{\eta^{2}}{h}\right)\right]+j \omega \sigma B_{0, x} \eta
$$

After some elementary integration, the correction terms (21) for $B_{y}$ and (22) for $B_{x}$ can be obtained. These expressions permit an iterative approach towards flux density values in the strand centres incorporating the effects of the eddy currents. Since the correction terms are very small for the strand configurations commonly applied, convergence is achieved after a few iterations.

$$
\begin{aligned}
& \Delta B_{y} \approx-j \omega \sigma \mu_{0} h B_{0, y} \frac{w^{2}}{4} \\
& \Delta B_{x} \approx-j \omega \sigma \mu_{0} w B_{0, x} \frac{h^{2}}{4}
\end{aligned}
$$

\section{Magnetic Slot Wedges}

Sometimes, magnetic slot wedges are employed in hydro generators. This is mainly for the reason of reducing slot ripple losses, Due to the resulting flux concentration in the wedge area, magnetic wedges have also slightly beneficial effects on the radial field losses because a larger portion of flux is diverted directly into the tooth and does not penetrate the uppermost strands. The inclusion of magnetic slot wedges is relatively straightforward by the theory of magnetic polarisation as detailed in [15]. The magnetic field within isotropic magnetised matter can be expressed via

$\mathrm{B}=\mu_{0} \mathrm{H}+\mathrm{P}=\mu_{0} \mathrm{H}+\mu_{0} \kappa \mathrm{H}=\mathrm{B}_{0}+\kappa \mathrm{B}_{0}$,

where $\mathbf{P}$ is the magnetic polarisation and $\kappa$ denotes susceptibility. The term $\mu_{0} \mathrm{H}$ is abbreviated by $\mathrm{B}_{0}$. The polarisation vector $\mathbf{P}$ can be thought of being generated by magnetic sources at the boundaries between magnetic material and free space. Unfortunately, the magnetic susceptibility $\kappa$ is non-linear. Therefore, a volume integral for the calculation of the field B originating from the magnetic wedge has to be applied.

$$
B_{0}^{\text {wedge }}=\mu_{0} H^{\text {wedge }}=\sum_{i} \int_{\Omega_{i}} \rho_{m, i} \nabla G d \Omega_{i}
$$

In (24), the volume integral over the wedge is be replaced by a sum over elementary volumes $\Omega_{i}$ within which the magnetic properties are assumed to be constant. The magnetic charge density is defined by $\mathbf{H}$ inside the wedge and the respective susceptibility. The resulting fields in any point can be calculated by the vector sum of $B_{0}^{\text {wedge }}$ and the fields originating from the walls and the conductors. Since $\kappa$ is dependent on the field values, an iterative process is necessary. The relative permeability of slot wedges is comparatively low. Consequently, the resulting convergence rates are favourably high.

\section{ANN Training}

Having found a BEM solution for the idealised slot, it is highly desirable to include other effects, such as the saturation of the stator teeth, into the estimations. Taking into account such effects in an analytical solution would yield expressions of high complexity. In order to circumvent this, a correction model based on a neural network of the Multi-Layer-Perceptron (MLP) type has been established. For a test set of 65 hydro generators, both a linear BEM-solution and the corresponding nonlinear FE results have been compared. Additionally, this has been repeated for different values of the slot temperature. A sample result in terms of flux densities can be seen in Fig. 9, whereas Fig. 10 depicts the electric losses in the individual strands. The flux density values of Fig. 9 have been scaled with respect to a hypothetical stray field at the slot opening, (25).

$B_{\text {scal }}=\mu_{0} \frac{\sum_{\text {all strands }}\left|I_{\text {strand }}\right|}{w_{\text {slot }}}$ 
In both figures, the $y$-position has been scaled by the slot depth. The increase of flux density can be seen clearly near the slot opening (i.e. $y \rightarrow 0$ in Fig. 9).

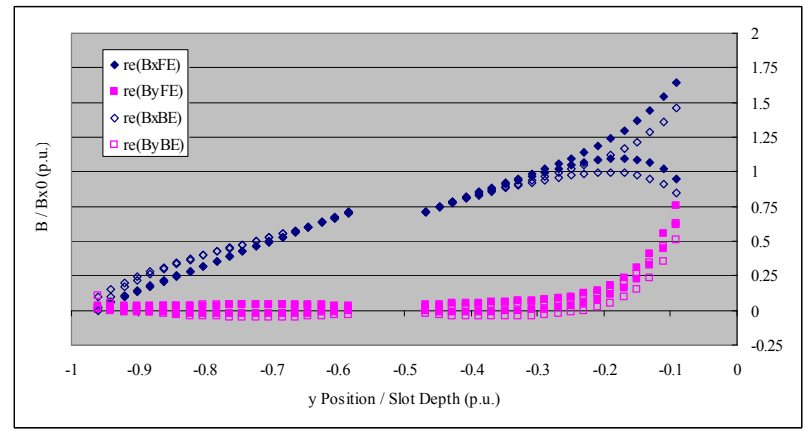

Fig. 9. Sample data set of the centres of the strands (BEM and FE solutions) for ANN training. Real part of $\mathbf{B}$ is shown.

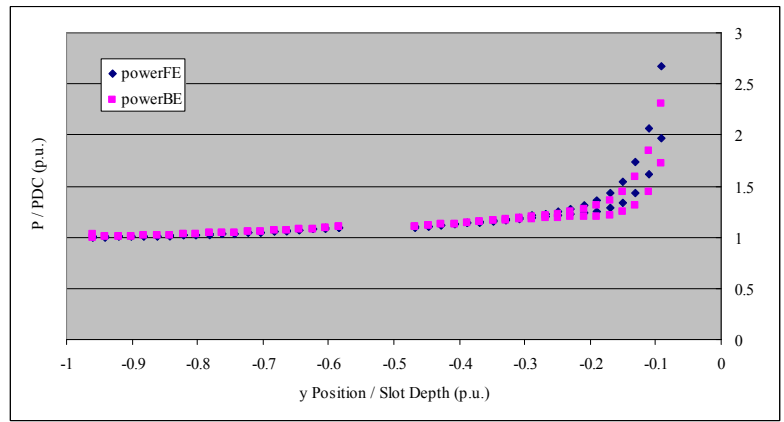

Fig. 10. Electric losses in the individual strands (BEM and FE results) scaled by the DC losses.

The values of the losses in the strands of Fig. 10 have been scaled by the DC losses. Ideally, all strands should exhibit only these losses. Moreover, the $x$-directed stray fields originating from the stator currents cause additional losses. This effect, however, is mitigated by the small strand height $h$. A $y$-directed fringe flux has better possibilities to generate losses since it penetrates the strands at their longer side (strand width $w$ ). This effect can be controlled by displacing the uppermost conductors away from the slot opening.

Comparisons between BEM and FE results clearly suggest a grey box approach. The solutions are not very far from each other, so constants $k_{i}$ for scaling the $x$ - and $y$-directed flux density at the strand centres and for the loss increase should suffice. These constants have been calculated for all $195(=65 \cdot 3)$ test cases and subsequently used as target vectors for training and validation. In order to prevent the net from learning the training vectors "by heart", the sets of training and validation vectors have been generated randomly. Additionally, to achieve better generalisation, Levenberg-Marquart training with Bayesian regularisation has been applied. The input of the net consists of normalised parameters such as frequency, conductivity, slot with and depth, strand dimensions, air gap flux, armature reaction etc. The training has been carried out with MATLAB's Neural Network Toolbox. For the approximation, an MLP-type network (Fig. 11) has been chosen.

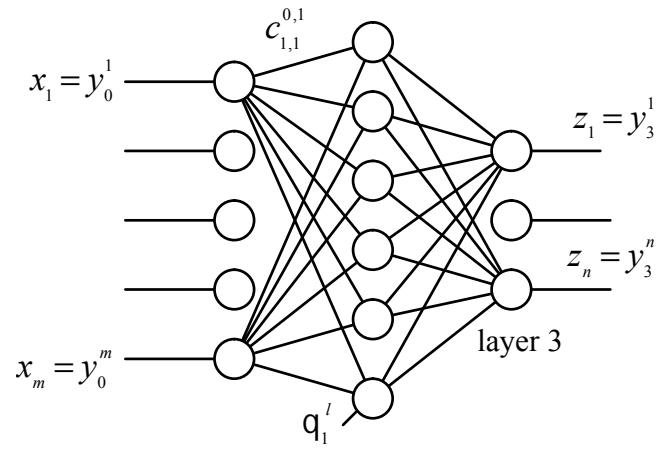

Fig. 11. Schematic representation of a three layer MLPnetwork. Not all connections have been drawn.

The output of a single neuron is computed in two stages. First, all input values and the threshold $\theta_{i}^{k}$ are summed. The inputs are obtained from the outputs $y_{i-1}^{j}$ of the preceding layer weighed by factors $c_{i, k}^{i-1, j}$. In the second stage, an activation function $f_{i}$ (e.g. sigmoid, hyperbolic tangent, or linear) is used to get the output value of the neuron.

$$
y_{i}^{k_{i}}=f_{i}\left(\theta_{i}^{k_{i}}+\sum_{j=1}^{k_{i-1}} c_{i, k_{i}}^{i-1, j} \cdot y_{i-1}^{j}\right)
$$

MLP networks are trained by backpropagation. That is, the error between network output and training vector is used to update the weight coefficients between the layers by a gradient method. Details can be found in any textbook on the method (e.g. [21]-[23]).

Having carried out the training, the network parameters are exported and used as an include file in a FORTRAN subroutine which implements the recall-phase of the trained network. This subroutine can be invoked in design programs at virtually no computational cost.

Unfortunately, finding the ideal network configuration is not straightforward. Whereas the neurons in the input and output layers are determined by the size of input and output vector, there exists some freedom in the formation of the hidden layers. However, these liberties are somewhat restricted. On the one hand, there should be enough neurons to capture the functional behaviour between input and output. On the other hand, overfitting should be avoided in any case, e.g. [25].

In order to assist with the choice of network parameters, a Genetic Algorithm (GA) has been employed to automatically investigate the parameter space for network creation (similar to the approaches in [23], [24]). For this sake, a GA Toolbox for MATLAB [19] has been used. The input values for the GA are the number of neurons in the hidden layers and the size of the training set. Accordingly, the rest of the data set has been applied for validation. Details of GAs can be found in [18]-[20], for example. The principle of a simple evolutionary method can be inferred from Fig. 12. After the generation of an initial population, the objective functions are computed and compared with the pre-defined optimisation goals. If 
these constraints could not be fulfilled, a new generation of individuals is formed by selecting the fittest members of the old population for a creation of offspring. These children are subsequently tested against the targets until either the conditions are met or the resources are exhausted.

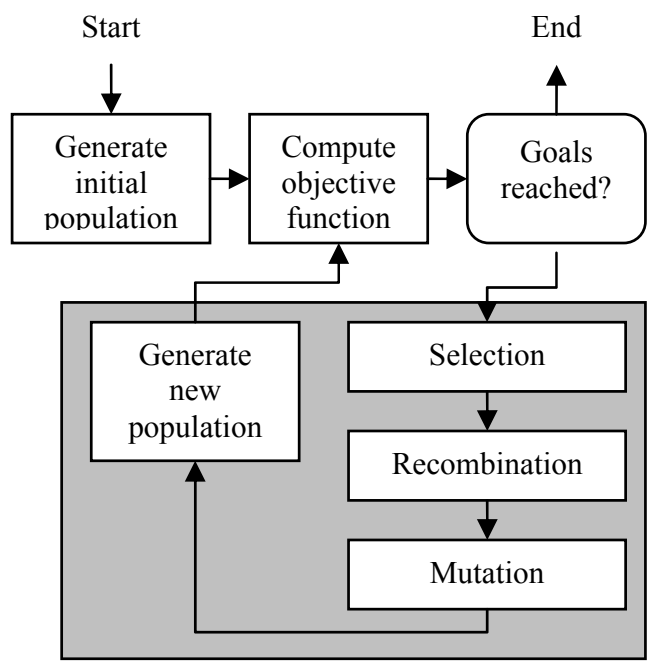

Fig. 12. Structure of a simple evolutionary algorithm, from [19].

In our case, the goal was to minimise a global mean error which had been computed from the relative deviations between network output and training vectors. From Fig. 13 , it can be deduced that there is a wildly fluctuating behaviour of this error during most of the GA's runtime. Amongst others, a reason for this behaviour is a random selection of training and validation vectors. Nevertheless, a certain amelioration of the situation is to be observed in the last quarter of the runs. Obviously, the GA has found a suitable combination of parameters that is robust enough to face the oddities of randomly chosen training vectors.

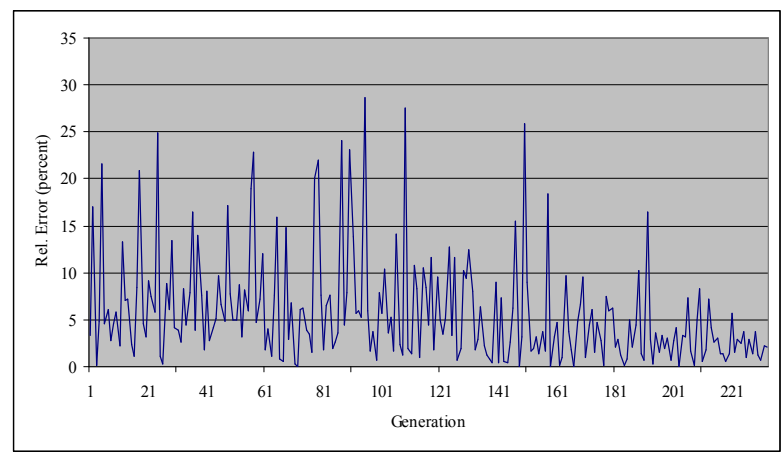

Fig. 13. Development of the global error during GA-based ANN-training.

A closer inspection of the error over the product of hidden neurons times training set size (Fig. 14) shows that there is a valley between 5000 and 6000 . Very low errors at the end of the abscissa indicate that the net has used nearly all vectors for training and hardly any for validation. This is not desirable for everyday use, since previously unseen input vectors might be misclassified. For that reason, it was decided to live with a somewhat higher global error instead of overtraining the network.

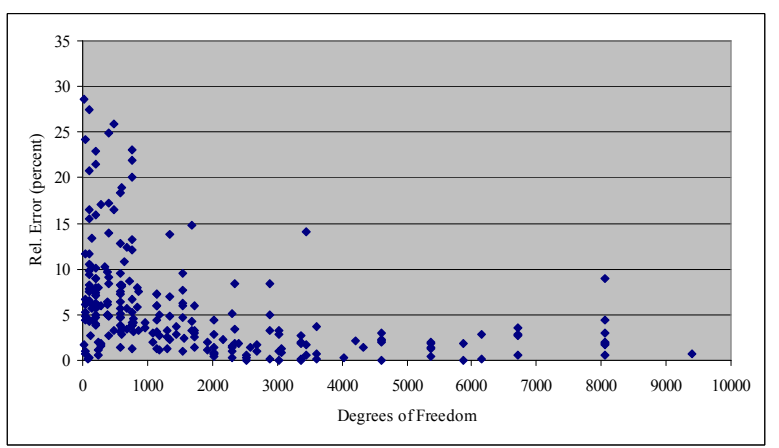

Fig. 14. Global error over number of neurons times number of training vectors.

Fig. 15, gives an impression of the recall phase of the trained network. There is relatively little deviation between training vectors and simulation by the network.

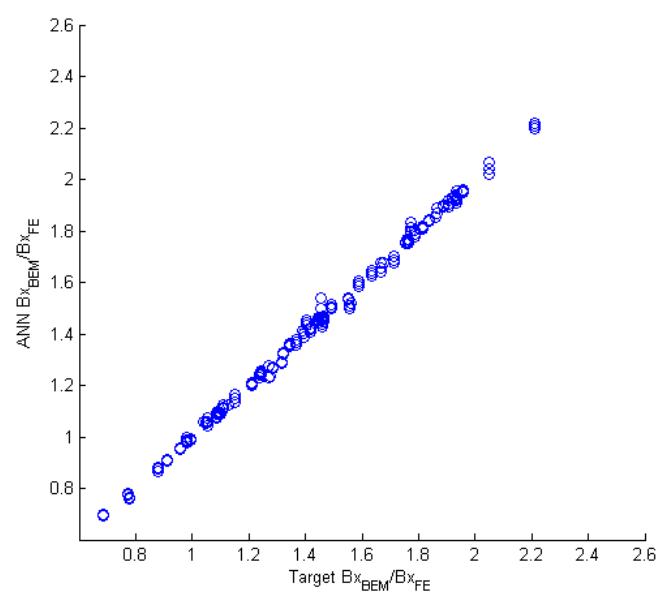

Fig. 15. Result of GA-based ANN-training for $B_{x}$.

\section{Results}

In Table I, the effects of tooth saturation and radial slot field on the additional losses in the slot region have been investigated for several generators. All losses are scaled by the DC losses in the slot region. Generator 1 is an example of a bulb type generator with highly saturated stator teeth and strands near the slot opening. Whereas the losses by the slot stray field $\left(P_{a d d}, B_{x}\right)$ are reduced by saturation, the radial field losses $\left(P_{a d d}, B_{y}\right)$ reach substantial values.

TABLE I. - Comparison of losses (percent of DC losses) in the slot region

\begin{tabular}{|c|c|c|c|c|c|}
\hline \multirow{2}{*}{ GEN. } & \multirow{2}{*}{ BAR } & \multicolumn{2}{|c|}{$B_{y}=0$, UNSAT. } & \multicolumn{2}{c|}{$B_{y} \neq 0$, SAT. } \\
\cline { 3 - 6 } & & $P_{a d d}, B_{x}$ & $P_{a d d}, B_{y}$ & $P_{a d d}, B_{x}$ & $P_{a d d}, B_{y}$ \\
\hline \multirow{2}{*}{1} & Top & 38.1 & 0.0 & 12.8 & 27.0 \\
\cline { 2 - 6 } & Bottom & 5.1 & 0.0 & 1.7 & 0.7 \\
\hline \multirow{2}{*}{2} & Top & 29.1 & 0.0 & 23.5 & 12.8 \\
\cline { 2 - 6 } & Bottom & 3.8 & 0.0 & 3.1 & 0.4 \\
\hline \multirow{2}{*}{3} & Top & 17.2 & 0.0 & 9.8 & 2.8 \\
\cline { 2 - 6 } & Bottom & 2.3 & 0.0 & 1.3 & 0.1 \\
\hline \multirow{2}{*}{4} & Top & 6.1 & 0.0 & 7.3 & 0.1 \\
\cline { 2 - 6 } & Bottom & 1.0 & 0.0 & 1.3 & 0.0 \\
\hline
\end{tabular}


Generator 2 is also a bulb type machine, albeit with lower saturation. Consequently, the saturation-based reduction of losses is lower than in the first case. Additionally to reduced saturation, a narrower slot leads to lower radial field losses compared to the first case. Generator 3 is a conventional vertical machine with moderate saturation in the stator teeth whereas generator 4 has several particularities. First, its tooth saturation is comparatively low. Therefore, the singularity of $B_{x}$ at the wedge bottom more than compensates the loss decrease due to saturation. Furthermore, the pre-slot is increased for short circuit reasons, which leads to a very small radial field at the uppermost strands.

Fig. 16 shows a comparison between the strand current distribution with and without $y$-directed slot field. As was to be expected, this influence can be neglected in slots with two columns of strands. However, this is not the case in turbine generators with double Roebel bars. This ongoing work will be presented in a separate paper.

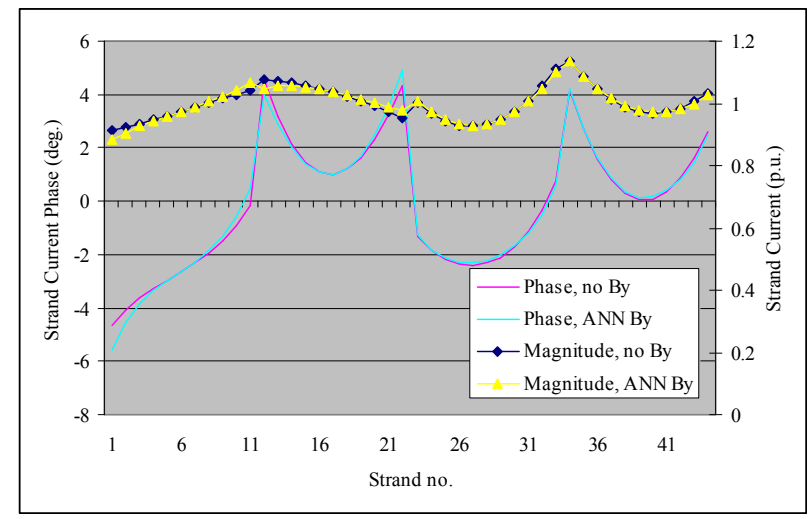

Fig. 16. Strand currents with and without $y$-directed slot field.

\section{Conclusions}

It has been demonstrated that a combination of linear Boundary Element results together with a neural network can provide fast approximate solutions to the slot opening problem for tendering and design purposes of hydro generators. Good estimations of the fields in the strands of generator stator bars are necessary for the calculation of radial field losses and circulating current distributions. Whereas the Boundary Element part takes care of the underlying physics, the neural network offers the possibility to learn second order effects such as tooth saturation from Finite Element test data.

\section{References}

[1] A. Schwery, G. Traxler-Samek, and E. Schmidt, „Numerical and Analytical Computation Methods for the Refurbishment of Hydro-Generators", Proc. of ICEM 2002, CD-ROM, Bruges, Belgium, 25-28 August 2002.

[2] G. Traxler-Samek, "Zusatzverluste im Stirnraum von Hydrogeneratoren mit Roebelstabwicklung", PhD-Thesis, Technische Universität Wien, 2003.

[3] J. Haldemann, "Transpositions in Stator Bars of Large Turbogenerators", IEEE Trans. Energy Conversion, vol. 19, no. 3, pp. 553-560, Sept. 2004.
[4] J. Haldemann, "Untersuchung verschiedener Verdrillungsarten in Statorwicklungen grosser Synchronmaschinen, deren Wirkung auf Strom- und Temperaturverteilung und Zusatzverluste in verschiedenen Betriebszuständen", PhD-Thesis, ETH Zürich, 1997.

[5] M. Iseli, K. Reichert, and G. Neidhöfer, „Calculation of Current Distribution and Stray Losses in Arbitrarily Transposed Stator Coils“, Proc. ICEM 1988, Pisa, Italy, pp. 47-52.

[6] M. Iseli,"Zusatzverluste in Statorwicklungen grosser Synchronmaschinen unter Berücksichtigung der Verdrillung und der Stirnraumfelder", PhD-Thesis, ETH Zürich, 1991.

[7] K. Oberretl, „Zusätzliche Wirbelstromverluste in Nutenleitern infolge eindringendem Luftspaltfeld“, Archiv für Elektrotechnik 60 (1978), pp. 121-127.

[8] W.-R. Novender, „Dreidimensionale nichtlineare Magnetfeldberechnung für Synchron-Turbogeneratoren zur Bestimmung der Schlingstromverluste in Roebelstäben", PhD-Thesis, Technische Hochschule Darmstadt, 1984.

[9] M. Dabrowski, A. Demenko, „Hybrid method for analysis of eddy-current loss in electrical machine winding caused by the main flux", IEEE Trans. Magn., vol. 24, no. 1, January 1988, pp. 479-82.

[10] O. Drubel, "Current distribution within multi strand windings for electrical machines with frequency converter supply", COMPEL, vol. 22, no. 4, 2003, pp. 1166-1181.

[11] C. Pozrikidis, A Practical Guide to Boundary Element Methods with the Software Library BEMLIB, Chapman \& Hall/CRC, Boca Raton (2002), pp. 265-269.

[12] A. Kost, Numerische Methoden in der Berechnung elektromagnetischer Felder, Springer-Verlag, Berlin (1994), pp. 194-224.

[13] G. Strassacker, P. Strassacker, Analytische und numerische Methoden der Feldberechnung, B. G. Teubner, Stuttgart (1993), pp.43-49, 92-105.

[14] K. Simonyi, Theoretische Elektrotechnik, $10^{\text {th }}$ ed., Barth Verlagsgesellschaft mbH, Leipzig (1993), pp. 97-117, 121-153, 307-316.

[15] H. Hofmann, Das elektromagnetische Feld, SpringerVerlag, Wien, 1974, pp. 254-271.

[16] F. Ollendorf, Berechnung magnetischer Felder, SpringerVerlag, Wien (1952), pp. 138-143.

[17] M. Markovic, M. Jufer, and Y. Perriard, „Analyzing an Electromagnetic Actuator by Schwarz-Christoffel Mapping", IEEE Trans. Magn., vol.40, no. 4, July 2004, pp. 1858-1863.

[18] Z. Michalewicz, D. B. Fogel, How to solve it: Modern Heuristics, Springer-Verlag, Berlin (2000), pp. 161-183, 341-346.

[19] H. Pohlheim, Evolutionäre Algorithmen, SpringerVerlag, Berlin (2000), pp. 7-71, 157-170.

[20] D. E. Goldberg, Genetic Algorithms in Search, Optimization, and Machine Learning, Addison Wesley Longman Inc., Reading, Ma. (1989), pp. 60-88.

[21] A. Zell, Simulation Neuronaler Netze, Addison-Wesley (D) GmbH, Bonn (1994), pp. 97-124.

[22] S. Haykin, Neural Networks, A Comprehensive Foundation, Macmillan College Publishing Company, New York (1994), pp. 138-220.

[23] A. Scherer, Neuronale Netze, Grundlagen und Anwendungen, Vieweg, Braunschweig (1997), pp. 71-91, 195-208.

[24] W. Wienholt, Entwurf neuronaler Netze, Verlag Harri Deutsch, Frankfurt/Main (1996), pp. 134-138.

[25] H. Demuth, M. Beale, Neural Network Toolbox, User's Guide, V. 4, The MathWorks, Inc., 2000, pp. 5.51-5.59. 